\title{
XLPE Cable Sheath Circulation Integrated Monitoring Terminal Design
}

\author{
Keqiang Sun ${ }^{1}$, Minghe Zou ${ }^{1}$, Baojun Wang ${ }^{2}$, Zhenhao Wang ${ }^{3}$ \\ ${ }^{1}$ State Grid LiaoNing Electric Power Company Limited Shenyang Power Supply Company, \\ LiaoNing Shenyang 110811, China \\ ${ }^{2}$ State Grid HeiLongjiang Electric Power Company Limited Jiamusi Power Supply Company, \\ HeiLongjiang Jiamusi 154002, China \\ ${ }^{3}$ Northeast Dianli University, Jilin Jilin 132012, China
}

Keywords: High voltage cables; Sheath circulation; Integration; Online Monitoring; Terminal

Abstract: In this paper, the problems about the practical application of cable sheath circulation online monitoring is analyzed, XLPE cable sheath circulation integrated monitoring terminal is designed, circuits design principles and parameters of monitoring terminal contains current transformer, circulation detection, wireless radio communications, auto-sensing power supply are introduced in detail, and applied to the actual project, to verify the reliability and usefulness of the integration of the monitoring terminal.

\section{Introduction}

Induced voltage is produced on metal sheath of XLPE cables, when the metal sheath and the earth formed passage, circulation generated on the metal sheath may reach higher values, which may cause sheath fever, lower capacity cable runs, etc. Metal sheath overheating will cause cable insulation aging, which is a threat to the safe operation of cable. Therefore, monitoring and analysis of the cable sheath circulation are needed, in present, the operating personnel usually get to the scene using clamp meter to obtain measured data, due to the grounding cable boxes scattered and large number of monitoring points, the complex tunnel environment, outdoor monitoring on base bar is also difficult, which brings a great deal of difficulties for circulation monitoring. Currently there are applications of circulation monitoring products, but in the application process encountered many problems, there is a lot of field data lines and communication lines, wiring is more difficult and does not conform to the requirements of electrical safety, what lines we monitor is above $66 \mathrm{KV}$, power can't be got directly, device power supply problems become critical, these issues need to be resolved in practice.

In this paper, an improvement and innovation combination on existing technology is taken, to overcome the deficiencies of the prior technology, using circulation monitoring integration, wireless communications, auto-sensing monitoring programs, to solve practical problems on monitoring high-voltage cable sheath circulation, provide a reasonable structure, high integration, without complicated wiring, simple and practical cable circulation integration monitoring terminal, especially for complex application environment of high voltage cable metal sheath circulation monitoring.

\section{Monitoring Terminal Design Plan}

Monitoring terminal is consist of retractable current transformers, microcontrollers, circulation detection circuit and other components. A retractable current transformer is dual-core and double-winding current transformer, in which one group of coil supply current detecting, the other is used for induction to take power. Cable circulation during signal processing of detecting circuit and is sent to $\mathrm{AD}$ conversion, then send it to CPU for data processing, and the measured data is sent to the remote data receiving device via radio frequency circuits. Power of all circuits is obtained by self-induced power taken circuit. The overall structure and principle block diagram are respectively shown in Fig. 1 and Fig. 2. In Fig.1, 1 is monitoring terminal microcontroller circuit, circulation detection circuit, wireless radio communication circuit, auto-sensing circuit to take part, integrated 
in a circuit board. 2 is open type current transformer, 3 is a fixed buckle of transformer.

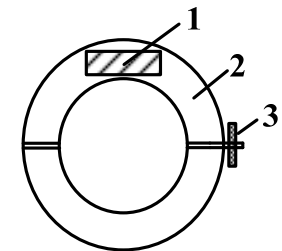

Fig. 1. Structure diagram

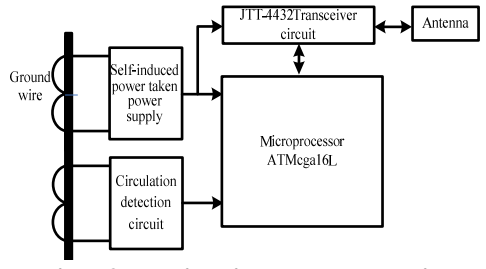

Fig. 2. Principle block diagram

\section{Monitoring Terminal Circuit Design}

Monitoring terminal board and open-type current transformer complete cable circulation data collection, storage, wireless transceiver as a whole and power supply of each unit.

\section{Microcontroller Circuit}

Microcontroller using Atmel's AVR microcontroller ATMEGA16L, with a high cost performance. ATMEGA16L's operating voltage is $2.7-5.5 \mathrm{~V}$, at a condition of $1 \mathrm{MHz}, 3.3 \mathrm{~V}$, the normal operating current is about $200 \mathrm{uA}$, idle operating current of $0.35 \mathrm{~mA}$, shown as Fig. 3.

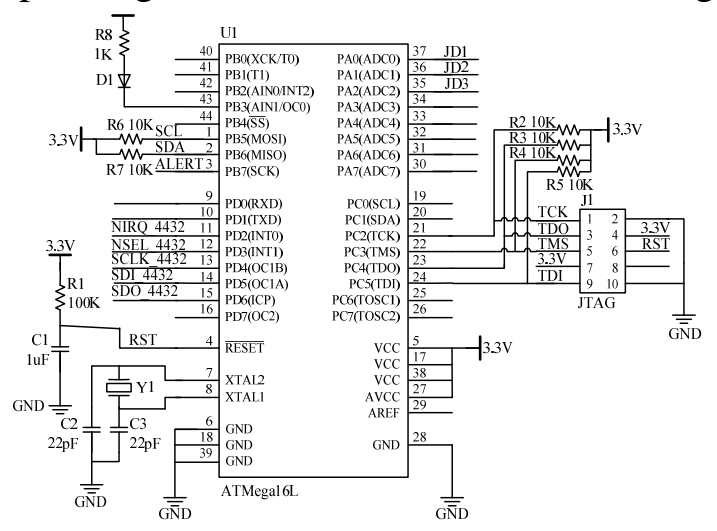

\section{Circulation Detection Circuit}

Fig. 3. Microcontroller and reset circuit

Circulation detection circuit of monitor terminal is consist of current transformers, operational amplifiers, $\mathrm{AD}$ analog-digital conversion, current transformer CT remove current through open type current transformer secondary winding, CT transformation ratio is $5 \mathrm{~A} / 2.5 \mathrm{~mA}$, turn into a voltage signal through a resistor and input to an emitter follower consist of operational amplifiers, then get it into $\mathrm{AD}$ analog-digital conversion circuit, convert to digital calculations send to the CPU, the measured data of cable circulation can be got. Principle circuit is shown in Fig. 4.

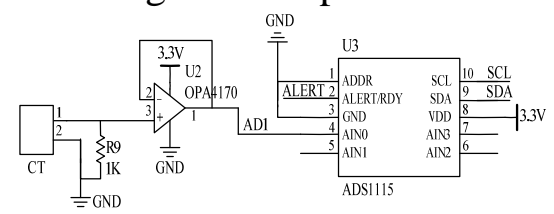

\section{RF Wireless Transceiver Circuit}

Fig. 4. Circulation detection circuit

High-performance radio frequency chip Si4432 and high-precision external components are used in the circuit. Module operating voltage is $1.9-3.6 \mathrm{~V}$, and works in $433 / 470$ / $915 \mathrm{MHz}$ ISM common band with modulator and demodulator, the data can be sent automatically packaged and received with automatically address match automatic CRC check. After the sending and receiving end, it will automatically set the NIRQ interrupt pin high level, to indicate transmit or receive completed, Si4432 provide a SPI interface to applied controller, and speed is determined by the microcontroller itself, so the programming is very convenient. Its power consumption is very low, the current is $85 \mathrm{~mA}$ when emit at an output power of $20 \mathrm{dBm}$, standby current is only $2.5 \mathrm{uA}$. Here are four operating modes of Si4432, down and SPI programming mode, standby and SPI programming mode, the reception mode, the transmission mode, the operating mode switch is achieved by configuration register 07H, shown as Fig. 5. 


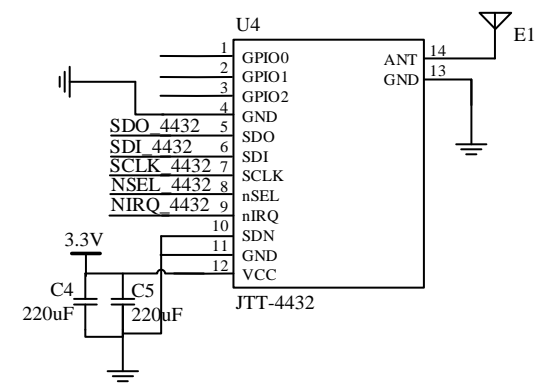

Fig. 5. Radio frequency communication circuitry

\section{Self-Induct Power Taken Power Supply Circuit}

Monitoring terminal power supply uses self-induct power taken pattern, socket on the cable grounding line through open type current transformer, a group of coil's secondary side in open type current transformer access to this circuit, the circuit is consist of retractable power taken CT, rectifier filter circuit, voltage protection and energy discharge circuit, transient protection, energy storage circuit, etc. Principle wiring diagram is shown in Fig. 6.

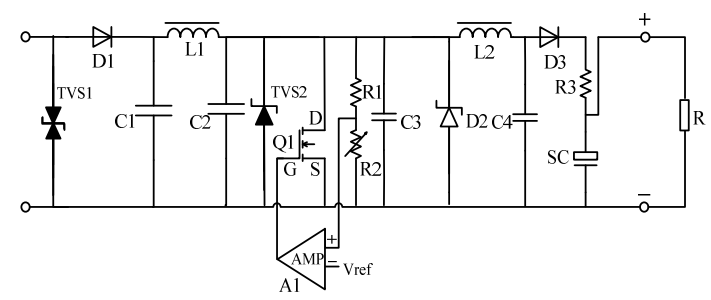

Fig. 6. Self-induct power taken power supply circuit

\section{(1) Rectifier Filter Circuit}

Retractable power taken CT secondary side connects to circuit, change AC into DC, through the rectifier filter consisted of D1, C1, C2 and L1.

\section{(2) Transient Protection}

When lightning or lines appear large short-circuit current, the court will induce a very high impulse voltage, add a bipolar TVS1 tube to the both sides of the coil which is before the rectifier filter circuit, to limit the impact of the output impulse voltage of induction coil. A single polarity rectifier filter circuit after termination TVS2 tube can guarantee that the follow-up circuit from the impact of impulse energy.

\section{(3) Voltage Protection and Energy Discharge Circuit}

After the rectifier filter, the voltage $U_{\mathrm{dc}}$ rise as current rise, after the iron core saturation, induction voltage also is higher. In order to protect the follow-up circuit, must be limit the voltage in the scope of technical requirements, use voltage protection and energy discharge circuit is shown in Fig. 7. When the voltage is greater than the set value, the voltage dividing resistor R2 is higher than the reference voltage Vref, voltage comparator LM393 outputs a high level, high-power MOS transistor is turned on, the excess energy exhausts through the power MOS tube; when Udc is low, the circuit does not work, it will not affect the power start-up current and low current work properly.

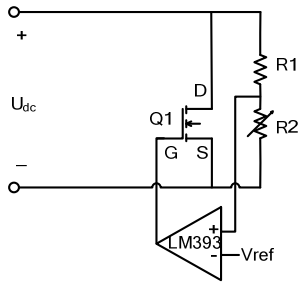

\section{(4) Storage circuit}

Fig. 7. Voltage protection and energy discharge circuit

Super capacitor SC of the circuit is used as energy storage element, problems of fast charge-discharge and low temperature environment can be solved, cable ground current monitoring device data transmission using wireless communication, which has a large instantaneous power of data transmission, the circuit has a large voltage drop, affecting the normal operation of other circuits, the join of super capacitor energy storage solves the problem of instantly large power 
supply. The power full account of various conditions of field using, it is possible to power supply stable and reliable in the case of low temperature and large current change.

\section{Conclusion}

This paper has designed XLPE cable sheath circulation integrated monitoring terminal, circuit design principles and circuit parameters are presented in detail, in the actual project site it has been verified. The terminal has features of reasonable structure, high integration, without complicated wiring, simple and practical, especially for complex application environments of high voltage cable metal sheath circulation monitoring.

\section{Reference}

[1] Aijun Zhu: EHV long and large cross-section cable sheath induced voltage. Power Supply, Vol. 23, No. 6 (2006), p. 7-9 (in Chinese)

[2] Xinrong Ni, Hongzhong Ma, Donghai Wang, et al: Techniques of voltage compensating and circulating current restraining of the metal shield for power cables. Automation of Electric Power Systems, Vol. 31, No. 5 (2007), p. 65-69, (in Chinese)

[3] Xiaoliang Zhuang, Zhaorong Yu, Haiqing Niu, et al: Experimental research of relationship between daily load factor and cyclic ampacity of $10 \mathrm{kV}$ XLPE cable. Electric Power Automation Equipment, Vol. 34, No. 4 (2014), p. 168-172 (in Chinese)

[4] Youyuan Wang, Rengang Chen, Weigen Chen, et al: Calculation of trench laying cable ampacity and its influencing factors. Electric Power Automation Equipment, Vol. 30, No. 11 (2010), p. 24-29 (in Chinese)

[5] Xutao Li, Kai Zhou, Li Wan, et al. Development of cable termination PD condition monitoring device based on TEV method. Power System Protection and Control, Vol. 41, No.12 (2013), p. 98-103 (in Chinese) 